\title{
Towards a self-structuring software library
}

\author{
H.Ye and B.W.N.Lo
}

\begin{abstract}
Software storage structuring and retrieval remain a major challenge to the widespread adoption of software reuse. An approach that can facilitate the automatic structuring of software components libraries is presented here. Based on the automatic indexing and the self-organising map (SOM) technologies, key features associated with software components can be identified and organised in a very simple way that makes their distance relations geographically explicit on the two-dimensional output layer of the SOM. The proposed approach was applied to a collection of UNIX commands to evaluate its retrival effectiveness. Preliminary results were encouraging and showed improvement on both recall and precision, with substantial reduction in the amount of human effort required in the process.
\end{abstract}

\section{Introduction}

Although the subject of software storage and retrieval has been investigated for over two decades, it still remains an active area of research in software reuse and software engineering. A number of solutions have been proposed, but the issue has not been satisfactorily resolved. Most existing solutions are either too ineffective to be useful or too intractable to be usable [1].

The purpose of this research is to propose a new and more efficient approach to building software libraries. A self-classification scheme is used to structure a software library, and a natural language based retrieval mechanism is provided to minimise human effort in locating desired software components from the library.

The most significant feature of software components is their functionality in terms of reuse purpose. Most software modules contain textual descriptions of their functions in the forms of system descriptions, operation manuals or user documents etc. Because the major interest in software retrieval is the functionality of the required components, these natural language documents can be used as the surrogates of the software components in the process of software classification and retrieval. The proposed classification scheme is based on software textual documentation rather than directly on the components themselves. Therefore the assumption is that 'good and reliable' documentation exists for the software components under consideration. If this were not the case, it would then be necessary to re-document the software before applying this method to build the desired software library. The task of how to construct or recover accurate functional descriptions for software is regarded as a separate issue and is not addressed in this paper.

\section{(C) IEE, 2001}

IEE Proceedings online no. 20010447

DOI: $10.1049 / \mathrm{ip}-\mathrm{sen}: 20010447$

Paper first received 28th May 1999 and in revised form 16th January 2001 H. Ye is with the Department of Computer Science and Software Engineering, The University of Newcastle, Callaghan, NSW 2308, Australia

E-mail: hye@cs.newcastle.edu.au

B.W.N. Lo is with the School of Multimedia and Information Technology, Southern Cross University, Lismore, NSW 2480, Australia E-mail: blo@scu.edu.au
Previous researchers [2-5] have suggested that the selforganising map (SOM) [6] is an ideal candidate for classifying textual documents. However, most SOMbased IR systems focus on document classification rather than direct retrieval. A retrieval system requires much more accurate clustering than a mere classification system. It is also reported that high recall and poor precision were observed in SOM-based document classification systems [4]. In software retrieval, the main purpose is not to retrieve all the documents in which a particular pattern exists but rather those best describing the desired component functionality. Thus, retrieval precision is more crucial in software retrieval than in typical IR domains (e.g. legal or medical domains).

In order to satisfy the above requirement in software retrieval, the proposed approach focuses on the accuracy, i.e. focuses on how to accurately represent the conceptual information implicitly existing in software documents and how to accurately classify the information.

The major contributions of the present research are:

- An indexing method, which is nearly completely automatic, is proposed to represent software documents. To ensure the accuracy of the subsequent SOM classification, it focuses on the quality of the indexing.

- A software self-organising map (SSOM) is developed to classify the documents. Some adaptations are made to improve the accuracy of the SOM-based clustering.

- The above two techniques, together with a natural language based retrieval mechanism, are integrated into a uniform model, on the basis of which a self-organising software library can be built.

The feasibility and effectiveness of the model have been demonstrated by the encouraging retrieval results obtained from an experiment using a prototype system.

This paper is concerned with the first two steps, software indexing and software self-organising map, while the natural language based retrieval mechanism will be considered in a future article.

\section{Review of previous research}

This section provides a brief review of previous research and the current status of software reuse libraries. It also 
discusses previous research based on SOM and automatic indexing.

\subsection{Current status of research in software reuse libraries}

A large amount of research effort on techniques for structuring software libraries for the storage and retrieval of software assets may be found in the literature. These techniques can be classified into three broad families: information science, artificial intelligence and hypertext [7]. The hierarchy of the classification and some relevant references are shown in Fig. 1.

Controlled vocabularies and knowledge-based techniques, in general, need intensive involvement of human efforts in manual indexing or knowledge acquisition and representation. The indexing task can be automated in uncontrolled vocabularies or free text indexing, as the index terms are often drawn directly from the text of the indexed objects. It may be a good alternative in reducing the high cost associated with manual indexing. But this kind of method is difficult to understand and interpret, as the semantic relationships between software components are often obscure. Mili et al. [1] recently presented a critical survey on software reuse libraries and defined a set of criteria to assess the methods. They concluded that while there is a wide range of solutions to this problem, none of them offers the right combination of efficiency, accuracy, user-friendliness and generality to facilitate a breakthrough in the practice of software reuse.

\subsection{SOM for organising textual information}

Ritter and Kohonen [2] first applied the SOM technology to textual analysis in an attempt to build semantic maps between words. These words and their context attributes were derived from 10,000 randomly generated artificial sentences. Nouns, verbs and adverbs are segregated into different domains on the map.

Lin et al. [3] used the SOM for classification on the 140 titles indexed by the descriptor 'Artificial Intelligence' in Silver Platter's CD-ROM version of the LISA database. The resulting semantic map suggests a list of document categories, and intuitively reveals the distribution of the documents.

More recently Orwig et al. [4] applied the SOM to the classification of electronic brainstorm output, and Honkela et al. [5] developed a WEBSOM method for exploring miscellaneous document collection in the World Wide Web.

The purposes of the above applications are all focused on document clustering, rather than document retrieval. It should be noted that the information used for classification is much less than that needed for retrieval because of the different requirements of the clustering. A fine-grained clustering is necessary to achieve high precision in a retrieval system whereas a coarse-grained clustering may be enough for a pure classification system.

Merkl et al. [16] developed a SOM for software document classification on a very small collection of 36 MSDOS commands. One of the major challenges of using SOM technology is how to index the objects being classified. However, this method used manual indexing, which is costly.

\subsection{Automatic indexing}

Salton [17] laid the theoretical foundation for automatic indexing. A set of single content identifiers can be recognised by their probabilistic term weighting, which is followed by a thesaurus transformation for the low frequency terms with small discrimination values, and a phrase transformation for the high frequency terms with negative discrimination values.

Maarek et al. [8] proposed a new automatic indexing scheme for software documentation based on the notions of lexical affinities and quantity of information. This technology has been implemented in the GURU system, which was used to construct an organised library of AIX utilities.

The approach described in this paper is based on the automatic free text indexing and unsupervised neural network technology, the SOM. The automatic free-text indexing method makes it more cost-effective than the controlled vocabularies and knowledge-based systems, while the SOM has the capability of providing the semantic relationships between software components, but the general free-text indexing systems do not. A small amount of human effort is introduced to improve the quality of the indexing. In the next few sections, the proposed approach will be described in greater detail.

\section{Input data representation for the SSOM}

SOM is an unsupervised neural network. No training data is required in the learning process. Instead, it learns from its input data. The learning result tends to approximate the probability density function of the input space. Thus, the accuracy of input data representation is crucial for the success of the SOM-based classification.

Previous applications based on SOM mentioned in Section 2 focused on 'classification', i.e. the goal of these applications was to get a set of categories under which the documents are classified. Because of the substantial number of documents, the categories are rela-

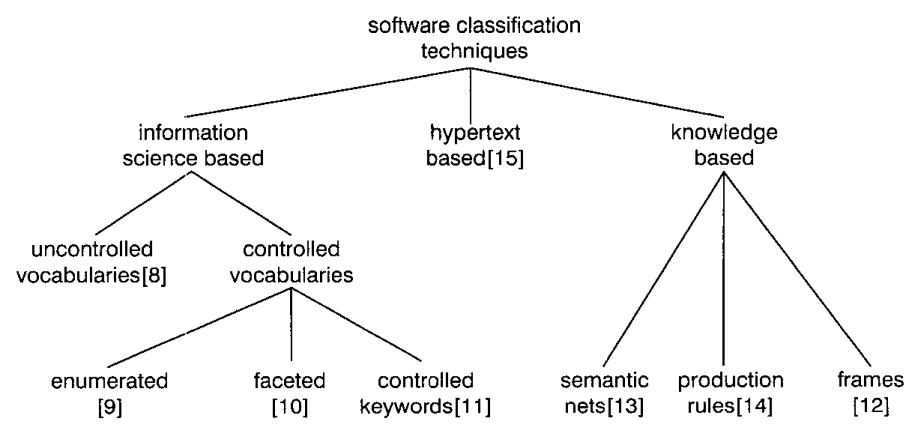

Fig. 1 Hierarchy of software classification techniques 
tively imprecise. In contrast, the ultimate goal of the SSOM is to locate desired software assets from the resulting map, i.e. it focuses on 'retrieval'. An imprecise categorisation is not good enough to ensure the level of precision for the retrieval. Therefore, a more elaborate indexing method is needed here.

To meet this requirement, a modified automatic indexing method [Note 1] is developed to extract features that are associated with each software document and assign each document a feature vector, which will be presented to the SSOM as input data. The features are special content identifiers characterising the documents from which they are identified. There is no syntactic and semantic analysis necessary in the indexing. Instead, the indexing process is based on the occurrence properties of the words within a document collection, called a corpus. In addition to single content identifiers, phrases that carry more specific semantic meanings than single terms are needed to promote retrieval precision, and a domain relational thesaurus that groups the semantically relevant or closely-related terms into term classes is created to enhance retrieval recall.

The major tasks involved in indexing are single term identification, phrase formation, and relational thesaurus construction. Based on the indexing results, feature vectors, or input vectors for the SSOM, can be obtained.

\subsection{Single term identification}

After removing all the common function words and stemming in a corpus, the best single content identifiers are those (1) occurring frequently in individual documents, and (2) occurring infrequently in the remainder of the collection. To measure the ability of an indexing term to uniquely distinguish a document in a corpus, Salton [18] introduced a parameter $w_{i j}$ (eqn. 1) called the weight of a term $T_{j}$ in document $D_{i}$.

$$
w_{i j}=t f_{i j} \cdot i d f_{j}=t f_{i j} \cdot \log \frac{n}{d f_{j}}
$$

Here $t f_{i j}$ is the term frequency for term $T_{j}$ occurring in document $D_{i}, d f_{j}$ is the document frequency defined as the number of documents in an $n$-document corpus containing $T_{j}$, and $i d f_{j}$ is the inverse document frequency which indicates how rarely a term $T_{j}$ occurs in the remainder of the corpus and is given by $\log \left(n / d f_{j}\right)$.

A threshold weight $w$ is then chosen. For each document $D_{i}$, all terms $T_{j}$ for which $w_{i j}>w$ are selected as single content identifiers and assigned to $D_{i}$.

\subsection{Phrase formation}

Phrases consisting of more than one word carry a more specific meaning than the separate single words. Phrases should not be randomly constructed. Terms with higher document frequency, called phrase heads, can be selected to form phrases. The phrase formation transforms higherfrequency, nondiscriminating terms into mediumfrequency phrases with greater discriminating power [18].

The following strategy is proposed to form phrases:

- Terms with higher document frequency than the threshold are selected as phrase heads.

- Combine the phrase head with its neighbouring words (ignoring the common function words), called phrase components, in the source document.

Note 1: It is not a 'complete' automatic method, as some human effort is involved in thesaurus construction (see Section 3.3). Because the amount of human effort is relatively small, it can still be regarded as automatic
indexing.

IEE Proc.-Softw., Vol. 148, No. 2, April 2001
- Calculate a phrase weight, which is the average weight of the phrase head and phrase component [19].

- Phrases with a higher phrase weight than a predetermined threshold are selected as indices.

\subsection{Relational thesaurus construction}

A thesaurus is a grouping of words or word stems, into certain subject categories called concept classes [17]. The terms in the thesaurus classes can replace the initial search terms and be used to increase retrieval recall. However, the concept classes may be diverse in different domains, and so generic thesauri will often not be specific enough for the text collection under consideration [20]. A domain dependent thesaurus is necessary for identification of the relationships among terms contained in a corpus.

The method used here is a semi-automatic method. Firstly, use a program to automatically build a termdocument matrix and calculate the term relatedness coefficient based on the term co-occurrence in the collection. Terms are content identifiers which have been identified at the previous stage. Next, a set of term pairs whose term relatedness coefficients are greater than the pre-determined threshold will be selected. Finally, the actual relationships between the terms will be recognised by domain experts. The human effort is restricted to a minimal level as the number of term pairs has been significantly reduced after the selection.

The above method has been applied to a collection of 97 UNIX command manual pages. After calculating the term relatedness coefficients, a threshold for the coefficient will be needed to select the term pairs. A low threshold may result in a more 'complete' thesaurus by selecting a larger number of term pairs. However, as the recognition of the term relationships involves human effort, too low a threshold may result in excessive human involvement. In addition, the size of the collection under consideration also influences the value of the threshold. The larger the size, the larger the threshold. After some experimenting, a threshold value of 3 appears to be a practical and reasonable choice in this small collection. Several hundreds of term pairs with a coefficient greater than the threshold are eligible for entering into the relational thesaurus. More details on relational thesaurus construction, and some concrete examples may be found in [21].

The following five term relationships were identified by a domain expert:

- Synonyms (S)

- Generic-specific (G-S)

- Whole-part (W-P)

- Modifier-modified (M-M)

- Irrelevant (I)

All (S) synonyms will be merged into one single term class, and the class, rather than individual terms, is used in the indexing and retrieval stages. The (GS) relationship represents a concept hierarchy. This makes it possible to classify specific topics under more general ones and to formulate a search request by starting with a general formulation and progressively narrowing the specification down to those areas that are of particular interest. The (WP) relationship includes two sub-relationships, attributive and constituent [22]. For example, name, mode, and owner are all attributes of file, while line and character are constituents of file. The (MM) relationship actually stands for a phrase which is based on the co-occurrence of the phrase elements in certain documents, rather than the adjacency of the phrase elements. These M-M pairs will be collected into the phrase thesaurus. The (I) term pairs will be ignored. 


\subsection{Weighted input vectors}

All the content identifiers extracted from a software document collection are regarded as the features associated with the whole software collection. The number of the content identifiers, or features, is the dimension of the input vector. Each element in a vector represents a certain feature. Each software component can then be schematically described by a vector, based on the presence or absence of the features that the component possesses.

In some systems, the vector describing the features of a document is assigned binary values. If a feature is present in a document, then 1 is assigned to the corresponding element of the vector, otherwise it is $0[2,3,16]$. It is argued that the binary vector is not sufficiently accurate for the software classification domain in the present research. After indexing, several features for each component have been recognised. Some features are significant in terms of the functionality of the software component, while the others may not be. It is expected that software components will be grouped on the output layer of the SSOM on the basis of the most significant features. Taking this into account, weighted input vectors are chosen rather than the binary ones. The 'extent of significance' of a feature can be indicated by the term weight obtained in the indexing stage, which can be directly used as the value of element in a weighted input vector.

Salton and Buckley [23] suggested that an appropriate term-weighting system is capable of enhancing retrieval effectiveness. The reason why weighting is so helpful will be illustrated by the results of two SOM simulations. Two SOMs were trained based on the two different kinds of input vectors, binary and the weighted input vectors (the training method will be introduced in Section 4). Both kinds of input vectors represent the same document collection, consisting of 97 UNIX manual pages. It was found that the weighted input vectors produced better clusters for this collection than did the binary input vectors. For example, the UNIX command sed is grouped with different commands by using different types of input vector. This is shown in Table 1. The column labelled 'Features' in Table 1 denotes on what basic feature(s) the corresponding commands are grouped. The features involved in Table 1 and their weights in different commands are listed in Table 2. An examination of the results in Tables 1 and 2 shows that using the weighted input vector gives a better and more meaningful group result. When the binary vector is used, sed can be grouped on the basis of all the features it has. There is no difference between the features in terms of the significance for the clustering. However, the main function of sed is editing text, thus its most important feature should be edit, which is indicated by the feature weight. When using the weighted input vector, the highly weighted feature edit makes it grouped with the commands ex and $\mathrm{vi}$ which also have the same highly weighted feature edit. It might be interesting to note that whatis is grouped

Table 1: Commands grouped with sed using different types of input vectors

\begin{tabular}{llll}
\hline \multicolumn{2}{l}{$\begin{array}{l}\text { Binary input vectors } \\
\text { Grouped }\end{array}$} & \multicolumn{2}{l}{ Weighted input vectors } \\
commands & Features & $\begin{array}{l}\text { Grouped } \\
\text { commands }\end{array}$ & Features \\
\hline $\mathrm{cp}$ & copy & $\mathrm{ex}$ & edit, output \\
tee & copy, output & $\mathrm{vi}$ & edit \\
whatis & edit, output & & \\
\hline
\end{tabular}

Table 2: Commands and their feature weights

\begin{tabular}{lllllll}
\hline Feature & \multicolumn{6}{l}{ Feature weight in different commands } \\
& sed & tee & cp & whatis & ex & vi \\
\hline Edit & 0.5279 & 0 & 0 & 0.3587 & 0.6021 & 0.4123 \\
Copy & 0.1706 & 0.1749 & 0.1699 & 0 & 0 & 0 \\
Output & 0.1499 & 0.3076 & 0 & 0.1238 & 0.3509 & 0 \\
Manual & 0 & 0 & 0 & 0.6109 & 0 & 0.1592 \\
\hline
\end{tabular}

with sed based on the feature edit using the binary input vector, but not grouped with sed even though what is has the same feature edit when a weighted input vector is chosen. From Table 2, it is seen that the feature edit is not the most highly weighted feature in whatis, instead, its most highly weighted feature is manual. Consequently, whatis should be classified mainly on the basis of the feature manual rather than the feature edit.

\section{The SSOM}

SOM can be viewed as a two-layer neural network, consisting of an input layer and an output layer. The input layer consists of a set of $n$-dimensional input vectors $\boldsymbol{x}_{i}=\left[\xi_{i 1}, \xi_{i 2}, \ldots, \xi_{i j}, \ldots, \xi_{i n}\right]^{T}$. Each input vector represents an indexed document. Here $n$ is the total number of features identified from the source documents, and $\xi_{i j}$ is a numerical value that denotes the weight of feature $j$ associated with document $i$. The output layer is a twodimensional grid consisting of a set of nodes, called artificial neurone. Each node on the grid is associated with an $n$-dimensional reference vector $\boldsymbol{w}_{i}=\left[\eta_{i 1}, \eta_{i 2}, \ldots\right.$, $\left.\eta_{i j}, \ldots, \eta_{i n}\right]^{T}$, whose dimension is the same as the input vector. During the learning process, input layer sends the input vectors onto the output grid. Each input vector is then compared in parallel with every reference vector associated with each node in the grid and mapped onto the bestmatching node, called the winning node, which has the smallest Euclidean distance (other metrics can be used as well) with the input vector.

The SOM has an internal learning rule that enables it to learn without any supervision. During learning, those nodes that are topographically close in the grid up to a certain geometric distance will activate each other to learn something from the same input vector. In other words, the reference vectors within the distance will be updated. This will result in a local relaxation or smoothing effect on the reference vector of neurones in this neighbourhood. After enough input vectors have been presented, the output nodes will be globally ordered in a natural way [24].

The major learning steps in SOM are (1) selecting winning nodes, and (2) updating reference vectors. To adapt this technology to the specific software classification environment, this research employs the Tanimoto similarity to select winning nodes and the lateral interaction rule to update reference vectors.

\subsection{Selection of the winning node}

As stated earlier, Euclidean distance $d(\boldsymbol{x}, \boldsymbol{w})$ (eqn. 2) is often used in selecting the winning node.

$$
d(x, w)=\|x-w\|=\sqrt{\sum_{i=1}^{n}\left(\xi_{i}-\eta_{i}\right)^{2}}
$$


In this section, we will demonstrate that Tanimoto similarity is a better choice because it provides a more accurate measure of the similarity of a vector pair under the context of the software document classification. Tanimoto similarity is one of a family of measures, widely used in IR, which give identical ranking for a set of vector pairs. Tanimoto similarity is defined as eqn. 3 :

$$
s(x, w)=\frac{(x, w)}{\|x\|^{2}+\|w\|^{2}-(x, w)}
$$

where $s(\boldsymbol{x}, \boldsymbol{w})$ is the Tanimoto similarity between vectors $\boldsymbol{x}$ and $\boldsymbol{w},(\boldsymbol{x}, \boldsymbol{w})$ is the dot product of $\boldsymbol{x}$ and $\boldsymbol{w},\|\boldsymbol{x}\|^{2}$ and $\|\boldsymbol{w}\|^{2}$ are the Euclidean norms of $\boldsymbol{x}$ and $\boldsymbol{w}$, respectively.

When an input vector $\boldsymbol{x}$ is presented to the grid, it will be compared in parallel with all the reference vectors associated with each node using eqn. 3. A node whose reference vector has the maximum similarity with $\boldsymbol{x}$ is selected as the winning node. Assume that its subscript is $c$, then:

$$
s\left(\boldsymbol{x}, \boldsymbol{w}_{c}\right)=\max _{j}\left(s\left(\boldsymbol{x}, \boldsymbol{w}_{j}\right)\right)
$$

The ultimate goal of software classification is to find functionally similar components. In this case, the emphasis is similarity rather than difference. For the two measures, the Euclidean distance emphasises difference, whereas the Tanimoto similarity favours similarity. Usually, the dimension of the features is very high due to the large number of components in a library collection. For an individual document vector, most of the feature elements are zero with a few nonzero. The decision whether a certain component is or is not a good candidate against a certain retrieval query depends on these few non-zero feature elements. It is the dot product in eqn. 3 that makes these nonzero values more significant than those in the Euclidean distance. The following example may help to illustrate this point.

Assume an input vector $\boldsymbol{x}$ will be compared with the two reference vectors $\boldsymbol{w}_{1}$ and $\boldsymbol{w}_{2}$ to decide which node is the winning node. Euclidean distance and Tanimoto similarity are used to compare the input vector and the reference vectors. The calculated results are presented in Table 3 . When the Euclidean distance is used node $e_{1}$ is the winner, but according to the Tanimoto similarity node ${ }_{2}$ should be superior to node $e_{1}$. It is obvious that the selection of node is much more reasonable than that of node ${ }_{1}$. Two common features exist in $\boldsymbol{x}$ and $\boldsymbol{w}_{2}$, but no common feature exists in $\boldsymbol{x}$ and $\boldsymbol{w}_{1}$. This is especially significant in the retrieval phase. At the beginning of the retrieval, the user queries are usually incomplete. If using Euclidean distance, the query vector may be mapped onto an irrelevant node, just like $\boldsymbol{x}$

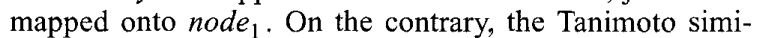
larity guarantees that the winning node possesses some similar features with the query vector, even though the query vector may not be complete. This conclusion is also supported by other research studies [25-27] where deter-

Table 3: Calculated Euclidean distance and Tanimono similarity between input vector and two reference

\begin{tabular}{|c|c|c|c|c|c|}
\hline \multicolumn{2}{|c|}{ Vectors } & \multirow[t]{2}{*}{ Measures } & \multirow[t]{2}{*}{$\boldsymbol{x}, w_{1}$} & \multirow[t]{2}{*}{$\boldsymbol{x}, \boldsymbol{w}_{2}$} & \multirow{2}{*}{$\begin{array}{l}\text { Winning } \\
\text { node }\end{array}$} \\
\hline$x$ & 00110000000 & & & & \\
\hline$w_{1}$ & 01000101000 & Eucidean distance & 2.236 & 2.449 & node $_{1}$ \\
\hline$w_{2}$ & 10110110111 & Tanimono similarity & 0 & 0.25 & node $_{2}$ \\
\hline
\end{tabular}
vectors

IEE Proc.-Softw., Vol. 148, No. 2, April 2001 minations of similarity between two vectors using the Tanimoto similarity yielded good results.

\subsection{The lateral interaction}

After a winning node is chosen, the node will learn from the input vector by updating its reference vector toward the input vector. The presented input vector affects not only the winning node, but also its neighbouring neurones. The degree of real neurone lateral interaction is usually described as having the form of a Mexican hat [6]. The interaction has a positive value between neurones with a small lateral distance and a negative value between neurones with a larger distance. As the lateral distance increases to a certain value, the interaction tends to 0 . The positive feedback is called lateral excitation, and the negative feedback is called lateral inhibition.

A similar effect should be reflected in the SOM learning process. After selecting a winning node, the nodes that are closest to the winning node should have a lateral excitation, i.e. their reference vectors will be adjusted toward the current input vector. The nodes that are less close to the winning node should have a lateral inhibition, i.e. their reference vectors will be adjusted away from the current input vector. Along with the increase of the lateral distance, when the interactions become zero, the reference vectors of these nodes will remain unchanged. This lateral interaction rule is mathematically described by eqn. 5 :

$$
I_{j}(t)=\left(A-l d_{c j} / \theta(t)\right) \cdot \exp \left(-B \cdot l d_{c j}\right)
$$

Here $I_{j}(t)$ is the lateral interaction of node ${ }_{j}, l d_{c j}$ is the lateral distance defined as the number of links or steps that must be taken from node to get to node $e_{j}$ under a certain topological structure. $A$ and $B$ are constants, where $A$ denotes the maximum positive interaction. $B$ is used to adjust the variation of lateral interaction. $A$ is set to 1 and $B$ is set to 0.4 in this research. $\theta(t)$ is a mono-decreasing function of time, used to adjust the range of the neighbourhood, which shrinks as time increases. It determines the turning point of the lateral distance at which positive interaction will become negative inhibition, and must be carefully chosen. If the neighbourhood is too small to start with, the map will not be ordered globally. On the other hand, if the neighbourhood is too large, the learning process will be difficult to converge. The SSOM starts the learning at a relatively large neighbourhood and then gradually decreases in size with the increase of time. The following equation is used to update reference vectors.

$$
\boldsymbol{w}_{j}(t+1)=\boldsymbol{w}_{j}(t)+\alpha(t) I_{j}(t)\left[x(t)-\boldsymbol{w}_{j}(t)\right]
$$

where $\boldsymbol{w}_{j}(t)$ is the reference vector associated with node at $_{j}$ time $t, \alpha(t)$ is the learning rate that decreases in time and converges to zero.

The lateral inhibition will make the convergence of the learning process faster since the establishment of the clusters is facilitated by the fact that nodes which are further away from the winning node are made even more dissimilar [16].

\section{Simulation of the SOM}

A prototype system was developed in accordance with the proposed SOM structure and learning algorithm, and a simulation on the system was conducted. 
Table 4: Some examples of indexing results

\begin{tabular}{|c|c|c|c|c|c|}
\hline $\begin{array}{l}\text { Is } \\
\text { Feature }\end{array}$ & weight & $\begin{array}{l}\mathrm{rm} \\
\text { feature }\end{array}$ & weight & $\begin{array}{l}\text { Ip } \\
\text { feature }\end{array}$ & weight \\
\hline Output & 0.26075 & remove & 0.73666 & request & 0.59666 \\
\hline Directory & 0.24018 & permission & 0.41174 & Ip & 0.39777 \\
\hline Permission & 0.23428 & write & 0.34833 & cancel & 0.33313 \\
\hline Character & 0.23280 & link & 0.34833 & print & 0.22585 \\
\hline Determine & 0.20756 & directory & 0.26381 & service & 0.22208 \\
\hline Sort & 0.20756 & file & 0.23603 & requestid & 0.22208 \\
\hline List & 0.18625 & symbolic & 0.23222 & associate & 0.19889 \\
\hline Format & 0.17790 & terminal & 0.19541 & status & 0.18243 \\
\hline Directory entry & 0.31899 & file remove & 0.60436 & standard & 0.17510 \\
\hline Character entry & 0.31531 & link remove & 0.40638 & print request & 0.41126 \\
\hline Column output & 0.27245 & write permission & 0.38003 & print service & 0.22397 \\
\hline Character specify & 0.23871 & directory permission & 0.33778 & print status & 0.20414 \\
\hline Determine output & 0.23415 & file write & 0.29218 & file print & 0.17452 \\
\hline Sort output & 0.23415 & symbolic link & 0.29027 & associate information & 0.17283 \\
\hline Format output & 0.21933 & standard permission & 0.26579 & & \\
\hline Directory list & 0.21321 & directory file & 0.24992 & & \\
\hline
\end{tabular}

\subsection{Simulation environment}

The prototype system was programmed in MATLAB and run on MS Windows 95. MATLAB is a powerful and comprehensive environment for performing technical computations. The present project is computationally intensive and involves many linear algebra and matrix computations. MATLAB has a strong support for this kind of computation.

A total of 97 manual pages of the most useful UNIX system commands [28] were chosen as the sample data of the simulation. Within the collection, 827 features are recognised. Some examples of the automatic indexing results are shown in Table 4.

\subsection{Self-classified software library}

$20 \times 20$ neurones on the output layer of the SSOM are arranged in physical positions according to a hexagonal topology. The input vectors are randomly presented to the SSOM. After 6000 learning iterations (taking about $5 \mathrm{~h}$ on a $400 \mathrm{MHz}$ PC), a classified UNIX command library was established, which is graphically shown in Fig. 2. Each dot

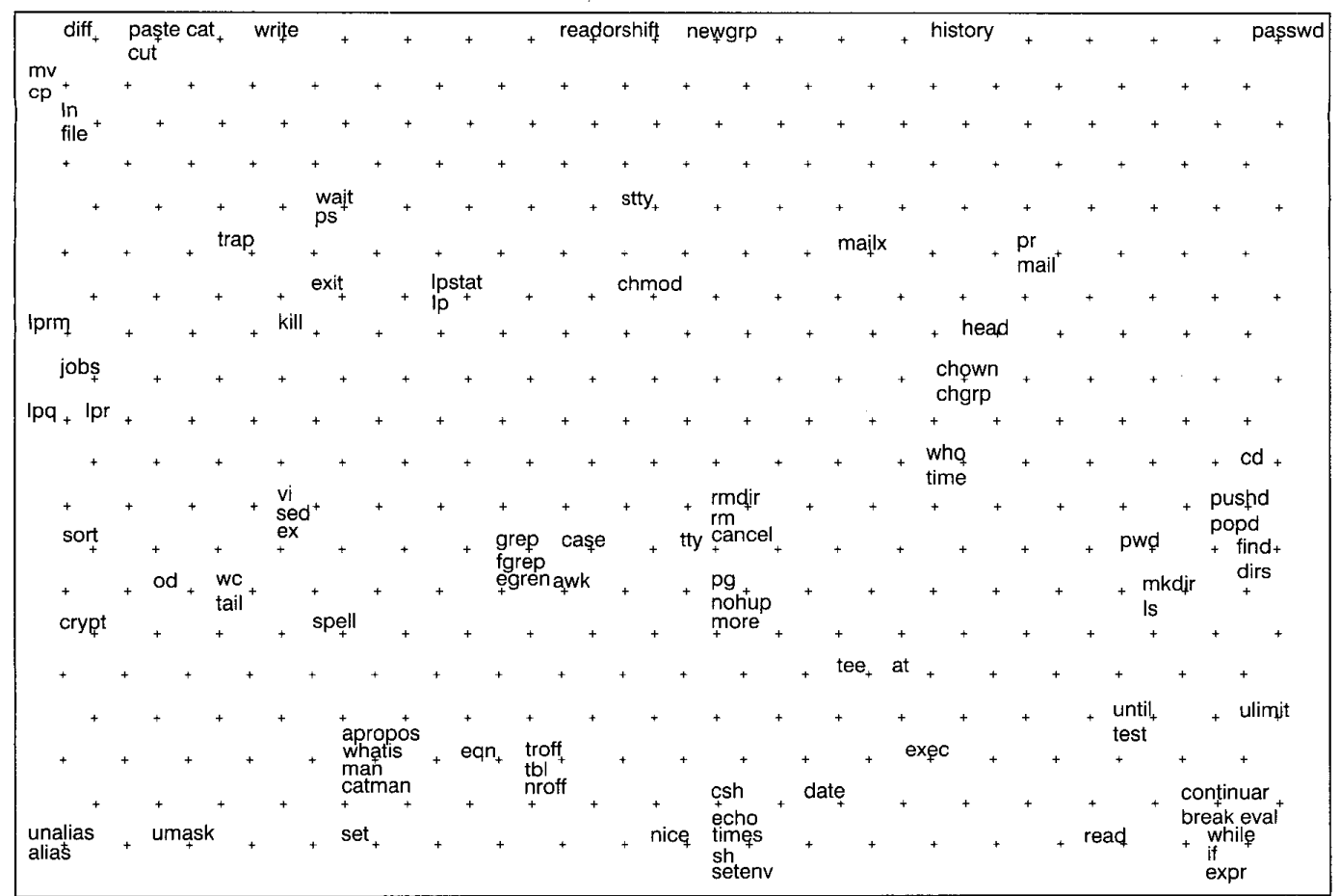

Fig. 2 Self-organising map of 97 UNLX command manual pages 
Table 5: Some component clusters found in Figure 2

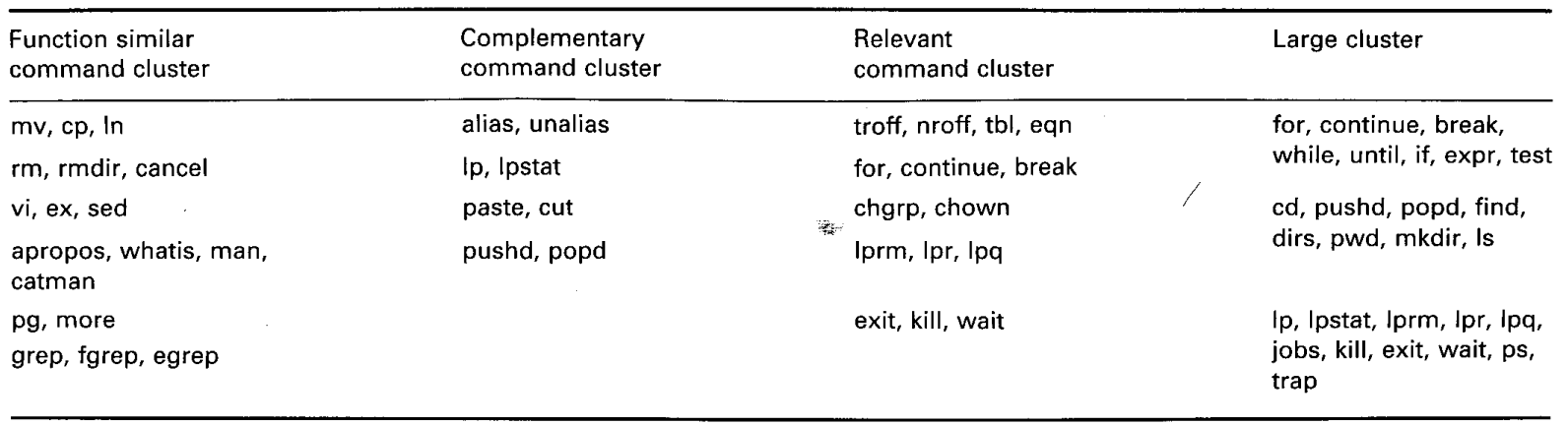

in Fig. 2 indicates a neurone. On the convergence of the SSOM, the winning neurones responding to the input vectors are marked with the name of the corresponding UNIX commands.

Some examples of the clusters shown in Fig. 2 are listed in Table 5. Under this classification scheme, there is no predefined category for the software components. The categories existing on the SSOM may be explained from different viewpoints. The examples of clusters shown in Table 5 may be one of the many possible explanations. There is no strict border for the categories, and the changes between the categories are smooth. This property makes it much more flexible than other classification schemes, reflecting the concept that software development is ever expanding and changing. Any rigid classification category would not be suitable to this ever-developing environment.

\subsection{Semantic meanings of the map}

In the SOM, only input stimuli are given to the network. No knowledge is supplied about what are the classifications, and what are the semantic meanings of those that the network derives. The final output of the SOM must be examined to assign semantic meanings and to determine the usefulness of the result [29].

The explanation of the semantic meanings of the SOM is very flexible. The feature relationships and clusters implicitly existing in the input space can be perceived on the map, which is characterised by the geographical adjacency of features or documents and therefore leaves much space for human recognition and imagination [3].

Fig. 3 may be one example of the many possible semantic explanations of the SSOM. As the SSOM converges, the values of the reference vectors have been updated in the learning process. To form the semantic map is to recognise which feature is most significant to a certain area, which may be determined as follows:

1) Select the most frequently occurring features to form a set of unit feature vectors (containing only one feature). Due to the high dimensionality of the feature space, it is impossible to map all features to the output layer. The features with higher frequency are considered the better representations of semantic relationships among the

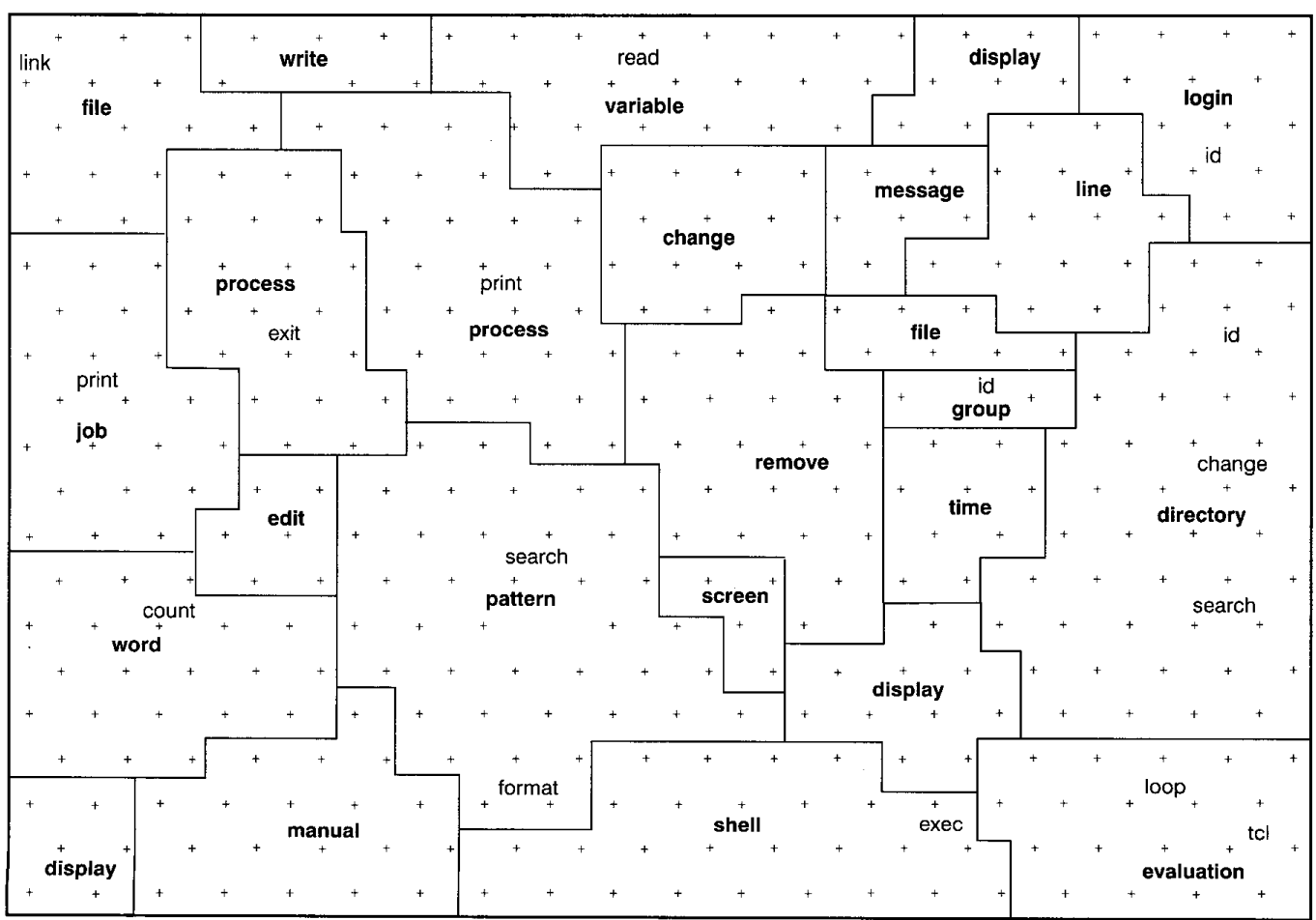

Fig. 3 Semantic map of the SSOM input feature space 
components. Only features occurring at least four times are selected.

2) Compare one reference vector to each unit feature vector in terms of Tanimoto similarity. The corresponding feature in the winning unit feature vector will be assigned to the node with which the reference vector is associated. 3) Repeat step 2, until all the nodes are assigned a feature.

Going through from step 2 to step 3, the most significant features to each node have been identified, and are shown in Fig. 3 in bold. Because the adjustments of the reference vectors toward a certain input vector occur in an adjacent area, the same feature is usually mapped to a continuous area. The boundaries clarify which feature belongs to which area. Some features of very high frequency in the collection, such as display, file may occur in more than one continuous area. The specific semantic meanings of these features in different areas depend on the features located in their neighbouring areas. For example, display in the rightupper corner of the map may indicate display message, whereas display in the lower left-hand corner of the map may indicate display manual.

Only some unit feature vectors become the winners in the first competition. Delete these winning unit feature vectors and let the remainder go through step 2 and 3 again, each node will be attached to another feature, which is shown in Fig. 3 as the non-bold letter. Therefore Fig. 3 is the overlap of the two map results (the multiple overlap also can be obtained by the same method to get a much more detailed semantic feature map).

Although Fig. 3 is only a two-overlapped semantic feature map, very rich information; such as the information about the functions of the components and relationships between the features, can be obtained from it.

If one superimposes Fig. 3 onto Fig. 2, and assumes that the figures are transparent, each component in Fig. 2 will belong to a certain feature area in Fig. 3, and its function can be explained by the feature(s) attached to the area. For example, a cluster, containing a set of UNIX commands: for, continue, break, while, until, if, expr, test, occurring in the lower right-hand area of Fig. 2 belongs to the same area in Fig. 3 containing the features of loop, TCl and evaluation. Most of the commands in the cluster are Tcl built-in looping commands, and all of them are relevant to the evaluation of the executable conditions.

The usefulness of the resultant semantic map may be viewed from another angle. To describe program functionality, Prieto-Diaz [30] defined a set of facets among which the most important facets are action and object. The pair, $\langle$ action, object $\rangle$, describes what a software component does. Action is the name of the specific primitive function performed by a component. 'Object' refers to the objects manipulated by the component. To create a set of keywords attached to each facet requires extensive human efforts. Another advantage of the semantic map is that a set of action-object pairs can often be recognised from the map as well. Some of the pairs are bound to the same area, e.g.

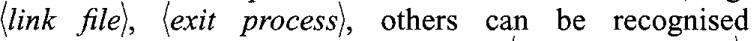
in adjacent areas in the map, e.g. (display message), (change process). More examples can be found in Table 6.

Some feature associations can also be obtained from Fig. 3. Relevant features seem to occur in adjacent areas. The following are some examples:

- display and screen: screen is the medium of display. - job and process: a process is usually part of a job under the UNIX context.

- login and id: login onto UNIX system needs a user id.
Table 6: Some action-object pairs identified in Fig. 3

\begin{tabular}{llll}
\hline $\begin{array}{l}\text { Pairs occurred in a same area } \\
\text { Action }\end{array}$ & $\begin{array}{l}\text { Pairs occurred in adjacent area } \\
\text { Action }\end{array}$ & Object \\
\hline Link & file & display & login \\
Read & variable & & line \\
Exit & process & message \\
Print & process & & variable \\
Print & job & manual \\
Remove & link & & word \\
Count & word & & directory \\
Search & pattern & & time \\
Format & pattern & change & message \\
Change & directory & & file \\
Search & directory & & process \\
Display & directory & & variable \\
\hline
\end{tabular}

- file and line: line is constituent of a file.

- message and line: line is also constituent of a message.

\subsection{Retrieval experiment}

A retrieval experiment based on the SSOM was conducted. Retrieval queries were described in natural language (English). These descriptions were transformed to query vectors by automatic indexing which is similar to the process of indexing software documents. Each query vector was mapped onto a winning node on the SSOM. Software components attached to the winning node and/or the nodes adjacent to the winning node will be selected as candidates for the specified query.

Queries used in this experiment were obtained from MSDOS. Under MS-DOS system, typing a command followed by the $/$ ? switch displays a screen of explanation on the command in terms of its functionality. It is considered that these queries are more objective as they were not specifically generated for this retrieval experiment. Thus the retrieval results would be more believable. Table 7 shows a set of descriptions of MS-DOS commands and their relevant UNIX commands included in this self-structuring software library. The relevance judgements were made by four UNIX system administrators who work in the Computer Help Desk at Southern Cross University and have an average five-year experience on UNIX system.

Each query vector is represented as an $n$-dimensional vector $\boldsymbol{q}_{i}=\left[\sigma_{i 1}, \sigma_{i 2}, \ldots, \sigma_{i j}, \ldots, \sigma_{i n}\right]$, where $n$ equals to the dimension of the feature space of the classified components. Each element of the query vector corresponds to the presence (denoted by ' 1 ') or absence (denoted by ' 0 ') of a certain feature included in the feature space. If all the features attached to a query are included in the feature space, then the query vector will be formed in an easy way, simply assigning 1 to the corresponding elements in the vector. Unfortunately, this is not always the case. The following steps may be used to deal with query features that are not included in the feature space:

- In addition to the domain synonym thesaurus, a public synonym dictionary WordNet [31] is used to find the synonymous relationships between the query features and component features.

- A rational thesaurus is provided to the user to find a better replacement in terms of the several relationships that are explained earlier. 
Table 7: Queries obtained from MS-DOS command descriptions and their relevant Unix components

\begin{tabular}{llll}
\hline Query No. & MS-DOS command & Descriptions & Relevant UNIX commands \\
\hline 1 & cd & displays the name of or changes the current directory & cd, pushd, popd, dirs, pwd \\
2 & copy & copies one or more files to another location & $\mathrm{cp}, \mathrm{mv}$ \\
3 & date & displays or sets the date & date \\
4 & del & deletes one or more files & $\mathrm{rm}, \mathrm{rmdir}$ \\
5 & edit & MS-DOS Editor & vi, ex, sed \\
6 & find & searches for a text string in a file or files & grep, egrep, fgrep \\
7 & mkdir & creates a directory & mkdir \\
8 & more & displays output one screen at a time & more, pg \\
9 & rmdir & removes (deletes) a directory & $\mathrm{rmdir}, \mathrm{rm}$ \\
10 & type & displays the contents of text files & cat, pg, more, tee \\
11 & xcopy & copies files and directory trees & $\mathrm{cp}$ \\
12 & dir & displays a list of files and subdirectories in a directory & is \\
\hline
\end{tabular}

- After the above refinement, some features that still fail to find related features included in the feature space would be discarded.

The formed query vectors were then presented to the SSOM. Fig. 4 depicts the mapped result. The winning nodes for each query vector are labelled with the name of the corresponding MS-DOS commands. It is easy to discover the relevant components in the structured library by superimposing Fig. 4 onto Fig. 2. Some components around each query winning node in Fig. 2 are considered candidates for the query. For example, the candidates for MS-DOS command find in Fig. 2 are grep, fgrep, and egrep, which are attached to the same node as the one the find command is attached to in Fig. 4. This is the exact match with a zero lateral distance between the query winning node and the node(s) that the candidates are attached. Some queries may not be directly mapped to the same node where the retrieval target resides. In this case, the retrieval target may be found on the enlarged retrieval area. For example, the retrieval target $c p$ for MSDOS command copy is located in the neighbouring node of the query winning node with a lateral distance of one.

The performance of the retrieval can be measured by recall and precision. Recall is the proportion of relevant material retrieved and precision is the proportion of retrieved material that is relevant. Table 8 depicts the recall and precision of the retrieval results in terms of the different lateral distances. The range of lateral distance is between 0 to 3 . For each query, recall(s) and precision(s) are calculated. If a calculated recall reaches 1 under a certain lateral distance, the calculation would be stopped even though the lateral distance is less than 3.

The retrieval result is encouraging. $75 \%$ ( 9 out of 12) of queries achieved a recall of 1 with zero lateral distance, in other words, they directly targeted the right nodes on the map. The average recall and precision with a lateral

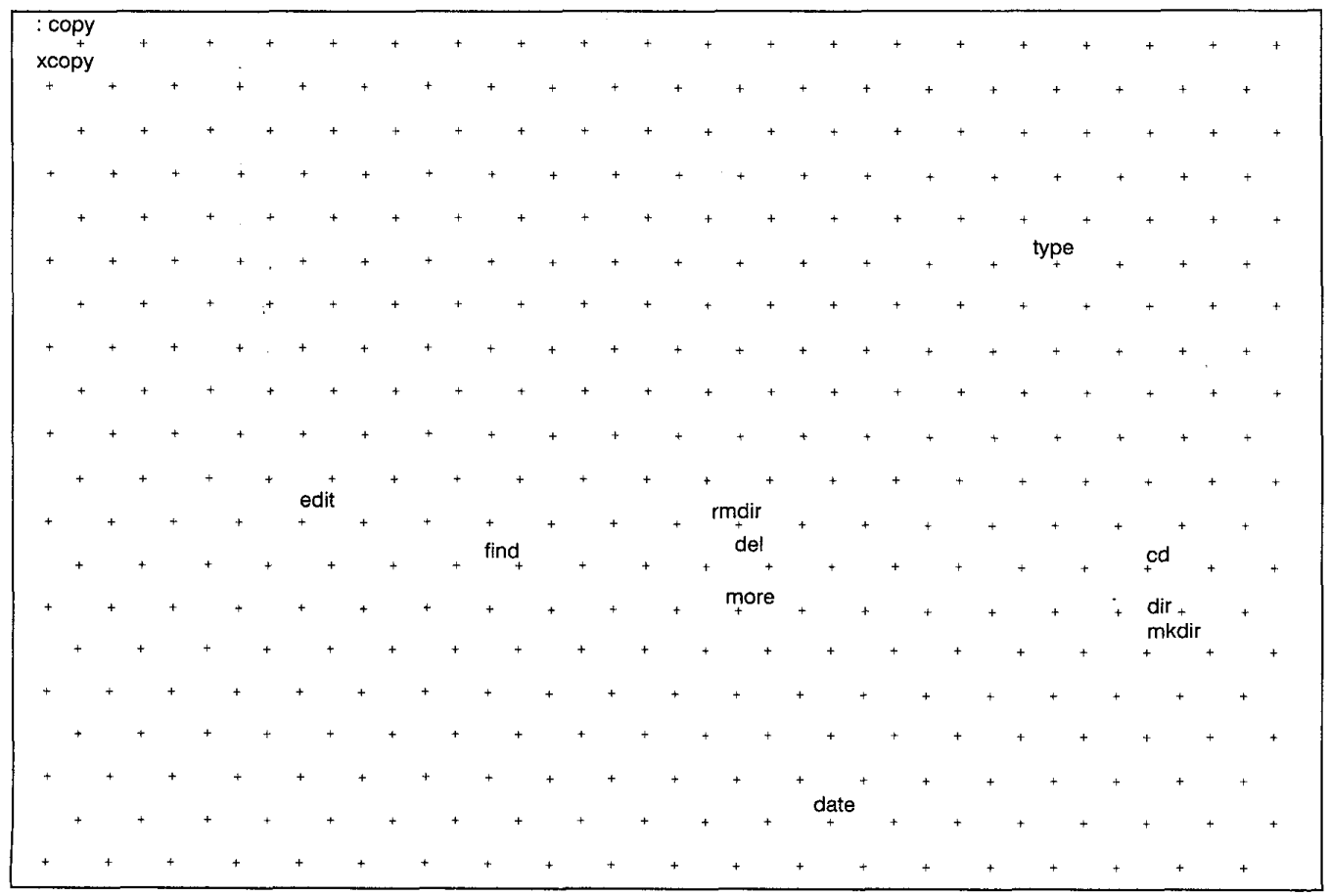

Fig. 4 Query map 
Table 8: Recall and precision measured under different lateral distances

\begin{tabular}{|c|c|c|c|c|c|c|c|c|c|}
\hline \multirow{3}{*}{$\begin{array}{l}\text { Query } \\
\text { No. }\end{array}$} & \multirow{3}{*}{$\begin{array}{l}\text { MS-DOS } \\
\text { command }\end{array}$} & \multicolumn{8}{|c|}{ Lateral distance } \\
\hline & & \multicolumn{2}{|l|}{0} & \multicolumn{2}{|l|}{1} & \multicolumn{2}{|l|}{2} & \multicolumn{2}{|l|}{3} \\
\hline & & recall & precision & recall & precision & recall & precision & recall & precision \\
\hline 1 & $\mathrm{~cd}$ & 0.2 & 1 & 0.2 & 0.333 & 0.8 & 0.571 & 1 & 0.625 \\
\hline 2 & copy & 0 & 0 & 1 & 0.4 & & & & \\
\hline 3 & date & 1 & 1 & & & & & & \\
\hline 4 & del & 1 & 0.667 & & & & & & \\
\hline 5 & edit & 1 & 1 & & & & & & \\
\hline 6 & find & 1 & 1 & & & & & & \\
\hline 7 & mkdir & 1. & 0.5 & & & & & & \\
\hline 8 & more & 1 & 0.667 & & & & & & \\
\hline 9 & rmdir & 1 & 0.667 & & & & & & \\
\hline 10 & type & 0.25 & 1 & 0.25 & 0.5 & 0.25 & 0.33 & 0.75 & 0.429 \\
\hline 11 & xcopy & 1 & 0.5 & & & & & & \\
\hline 12 & dir & 1 & 0.5 & & & & & & \\
\hline
\end{tabular}

distance of zero are 0.792 and 0.75 , respectively, in other words, more than $70 \%$ of queries achieved high precision at a high level of recall.

In this retrieval experiment, if queries directly target the right node and all relevant components are attached to the node, a recall of 1 is achieved. In this case, it is impossible to obtain a value of precision at a lower level of recall. In order to compare with other retrieval methods, precision needs to be measured at different levels of recall. To achieve this, retrieval results shown in Table 8 were regulated by using the following strategies:

- If more than one value of precision has been obtained at a given level of recall, the average value of precision is used at that level.

- If there is no value of precision at a certain recall level, then the precision is considered as the same as that at the next nearest higher level of recall.

Generally speaking, high recall results in low precision. This regulation is conservative, therefore it is acceptable. The regulated result is shown in Table 9.

The same test collection and queries were also applied to a public retrieval system - personal librarian (PL). PL is

Table 9: Regulated retrieval results

\begin{tabular}{lllll}
\hline Query No. & Recall & & & \\
& 0.25 & 0.5 & 0.75 & 1 \\
\hline 1 & 0.665 & 0.571 & 0.571 & 0.625 \\
2 & 0.4 & 0.4 & 0.4 & 0.4 \\
3 & 1 & 1 & 1 & 1 \\
4 & 0.667 & 0.667 & 0.667 & 0.667 \\
5 & 1 & 1 & 1 & 1 \\
6 & 1 & 1 & 1 & 1 \\
7 & 0.5 & 0.5 & 0.5 & 0.5 \\
8 & 0.667 & 0.667 & 0.667 & 0.667 \\
9 & 0.667 & 0.667 & 0.667 & 0.667 \\
10 & 0.61 & 0.429 & 0.429 & 0.041 \\
11 & 0.5 & 0.5 & 0.5 & 0.5 \\
12 & 0.5 & 0.5 & 0.5 & 0.5 \\
Average & 0.681 & 0.658 & 0.658 & 0.631 \\
\hline
\end{tabular}

54

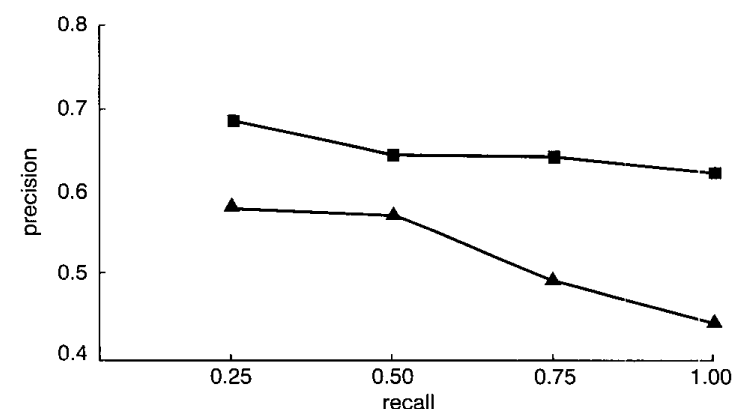

Fig. 5 Comparison of recall and precision

SSOM

$\Delta \mathrm{PL}$

one of the PLS products for building and searching fulltext databases. Comparison of recall and precision between the SSOM and PL is shown in Fig. 5. This comparison shows that SSOM achieved a higher precision at the same level of recall than did PL. A $25.4 \%$ improvement of average precision under different levels of recall was observed.

\section{Conclusion}

An approach to building software libraries has been presented. The approach has been implemented and assessed by a prototype system and a simulation on the system. 97 manual pages of the most useful UNIX system commands were chosen as the sample data in the simulation. A self-structuring library containing the commands is presented. A retrieval experiment was carried out on a set of natural language queries. The retrieval result shows that the SSOM has a better comprehensive retrieval performance in comparison with another public retrieval system (viz. PL).

This approach presents clear advantages in terms of cost, expandability and ease of visualisation in building a software library.

- Low cost and high efficiency: Because automatic techniques are used in both indexing and classification, the amount of required human efforts has been significantly reduced. 
- Expandability: SSOM can accommodate a continually expanding collection of software components, which is an inevitable process in most software organisations.

- Visualisation: The resulting map lends itself readily to visualisation, and thus the semantic relationships amongst components can be illustrated in a very intuitive manner. This would make it much more user-friendly than some other software libraries. Hypertext techniques could be used to construct browsing links based on the map.

The proposed approach is an IR-based method, therefore, it can only be used in situations where software documentation is available. The retrieval result presented in this paper is viewed as a preliminary test result. This approach will be further evaluated based on larger collections and query sets in future.

\section{Acknowledgment}

We would like to thank the referees for their valuable comments and constructive suggestions.

\section{References}

1 MILI, A., MILI, R., and MITTERMEIR, R.T.: 'A survey of software reuse libraries', Ann. Soft. Eng., 1998, 5, pp. 349-414

2 RITTER, H., and KOHONEN, T.: 'Self-organizing semantic maps', Biol. Cybern., 1989,61 , pp. 241-254

3 LIN, X SOERGEL, D., and MARCHIONINI, G. "A self-organizing semantic map for information retrieval'. The Fourteenth annual International ACM/SIGIR conference on Research and development in information retrieval, 1991, Chicago, IL, pp. 262-269

4 ORWIG, R.E., CHEN, H., and NUNAMAKER, J.F.: 'A graphical, selforganizing approach to classifying electronic meeting output', $J . \mathrm{Am}$. Soc. Info. Sci., 1997, 48, (2), pp. 157-170

5 HONKELA, T., KASKI, S., LAGUS, K., and KOHONEN, T.: 'Newsgroup exploration with WEBSOM method and browsing interface'. Technical Report, Report A32, 1996, Helsinki University of Technology, Helsinki

6 KOHONEN, T.: 'Self-organization and associative memory' (SpringerVerlag, Berlin, 1988), 2nd edn

7 FRAKES, W.B., and GANDEL, P.B.: 'Representing reusable software', Info. Soft. Technol., 1990, 32, (10), pp. 653-664

8 MAAREK, Y.S., BERRY, D.M., and KAISER, G.E.: 'An information retrieval approach for automatically constructing software libraries', IEEE Trans. Softw. Eng., 1991, 17, (8), pp. 800-813

9 PALMER, C. 'Camp undate'. Proceedings of AIAI computers in aerospace VII, American Institute of Aeronautics and Astronautics, 1989 , space VII, American I
Washington, DC, USA

10 PRIETO-DIAZ, R.: 'A software classification scheme'. Doctoral Disser- tation, Department of Computer and Information Science, University of California at Irvine, 1985

11 ARNOLD, S., and STEPOWAY, S.: 'The ReuseSystem: Cataloguing and retrieval of reusable software', in 'Software reuse: Emerging technology' (IEEE Computer Society Press, 1988)

12 ALLEN, B., and LEE, D.: 'A knowledge-based environment for the development of software parts composition systems'. Proceedings of 11 th International Conference of Software engineering, 1989, Pittsburgh, PA, USA

13 SOLDERITSCH, J., WALLNAU, K., and THALHAMER, J. 'Constructing domain-specific Ada reuse libraries'. Proceedings of 7th Annual National conference of Ada technology, 1989, USA

14 BOLLINGER, T., and BARNES, B.: 'Reuse rules: An adaptive approach to reusing Ada software'. Proceedings of AIDA 88, 1988, George Mason University, Fairfax, VA, USA

15 ISAKOWITZ, T., and KAUFFMAN, R.J.: 'Supporting search for reusable software objects', IEEE Trans. Softw. Eng., 1996, 22, (6), pp. $407-423$

16 MERKL, D., TJOA, A.M., and KAPPEL, G.: 'Learning the semantic similarity of reusable components'. Third International conference on Software reuse, 1994, Rio de Janeiro, Brazil, pp. 33-41

17 SALTON, G.: 'Automatic information organization and retrieval' (McGraw-Hill Book Company, New York, 1968)

18 SALTON, G.: 'Automatic text processing: The transformation, analysis, and retrieval of information by computer' (Addison-Wesley Publishing Company, MA, 1989)

19 FAGAN, J.L.: 'The effectiveness of a nonsyntactic approach to automatic phrase indexing for document retrieval', J. American Soc. Info. Sci. $1989,40,(2)$, pp. 115-132

20 SCHUTZE, H., and PEDERSEN, J.O.: 'A co-occurence-based thesaurus and two applications to information retrieval', Info. Proc. Manage. 1997, 33, (3), pp. 307-318

21 YE, H., and LO, B.W.N.: 'Integrating automatic indexing and feature competitive algorithm to represent input data for self-organizing map'. Technical Report, SOMTT 99/01,1999, School of Multimedia \& Information Technology, Southern Cross University, Lismore, Australia

22 CASAGRADNDE, J., and HALE, K.: 'Semantic relations in Papago folk definitions'. Studies in Southwestern Ethnolinguistics, 1967 Hague, Nertherlands, pp. 165-193

23 SALTON, G., and BUCKLEY, C.: 'Term-weighting approaches in automatic text retrieval', Info. Proc. Manage., 1988, 24, (5), pp. 513-523

24 KOHONEN, T.: 'Self-organizing maps' (Springer-Verlag, Berlin, 1997), 2nd edn

25 MINKER, J., PELTOLA, E., and WILSON, G.A.: 'Tech. Report' Report 201, 1972, Computer Sci. Centre, College Park, MD, University of Maryland

26 SPARCK-JONES, K.: 'Automatic keyword classification and information retrieval' (Butterworth, London, UK, 1971)

27 LIENARD, J., MLOUKA, M., and MARIANI, J.: In preprints of the speech communication seminar (Almqvist \& Wiksell, Uppsala, Sweden, 1975)

28 CHRISTAIN, K.: 'UNIX command reference guide' (John Wiley \& Sons Inc., New York, 1987)

29 TURBAN, E.: 'Expert system and applied artificial intelligence' (Macmillan Publishing Company, New York, 1992)

30 PRIETO-DIAZ, R, and FREEMAN, P.: 'Classifying software for reusability', IEEE Softw., 1987, 4, pp. 6-16

31 MILLER G A BECKWITH, R FELLBAUM, C., GROSS, D and MILLER, K.: 'Five papers on WordNet'. CSL Report 43, 1990 Cognitive Science Laboratory, Princeton University 